\title{
Preliminary Analysis Of ISS Maintenance History And Implications For Supportability Of Future Missions
}

\author{
J. Kevin Watson ${ }^{1}$ and William W. Robbins ${ }^{2}$ \\ 'Advanced Development Office, NASA-Lyndon B. Johnson Space Center, Houston TX 77058 \\ ${ }_{2}^{2}$ International Space Station Program Office, NASA-Lyndon B. Johnson Space Center, Houston, TX 77058
}

Contact Author:281-483-0971, j.k.watson@nasa.gov

\begin{abstract}
The International Space Station (ISS) enables the study of supportability issues associated with longduration human spaceflight. The ISS is a large, complex spacecraft that must be maintained by its crew. In contrast to the Space Shuttle Orbiter vehicle, but similar to spacecraft that will be component elements of future missions beyond low-Earth orbit, ISS does not return to the ground for servicing and provisioning of spares is severely constrained by transportation limits. Although significant technical support is provided by ground personnel, all hands-on maintenance tasks are performed by the crew. It is expected that future missions to distant destinations will be further limited by lack of resupply opportunities and will, eventually, become largely independent of ground support.

ISS provides an opportunity to begin learning lessons that will enable future missions to be successful. Data accumulated over the first several years of ISS operations have been analyzed to gain a better understanding of maintenance-related workload. This analysis addresses both preventive and corrective maintenance and includes all U.S segment core systems. Systems and tasks that are major contributors to workload are identified. As further experience accrues, lessons will be learned that will influence future system designs so that they require less maintenance and, when maintenance is required, it can be performed more efficiently. By heeding the lessons of ISS it will be possible to identify system designs that should be more robust and point towards advances in both technology and design that will offer the greatest return on investment.
\end{abstract}

